\title{
ON HAIRPIN VORTICES IN A TRANSITIONAL BOUNDARY LAYER
}

\author{
Ondřej HLADíK, Pavel JONÁŠ, Václav URUBA
}

\begin{abstract}
In the presented paper the results of experiments on transitional boundary layer are presented. The boundary layer was generated on smooth flat wall with zero pressure gradient forming one side of the channel of rectangular cross section. The hairpin vortices, packets of hairpin vortices, turbulent spots and calmed regions were experimentally investigated using time-resolved PIV technique.
\end{abstract}

\section{INTRODUCTION}

The transition from laminar to turbulent flow is of great practical interest. The final phase of laminar boundary layer transition starts always with the occurrence of first turbulent spots. Emmons [1] first reported the turbulent spots or simply spots as isolated regions of strong fluctuations that are streamwise carried, growing in size and coalescing with neighbours within the transitional boundary layer. The hairpin vortices and packets of hairpin vortices are typical structures within turbulent spots. Spots appear irregularly in time and at arbitrary location of the boundary layer and they are considered to be the building blocks of boundary layer turbulence, they control the length of the transition region etc. - see e.g. Narasimha [2]. The turbulent spots followed by calmed regions are defined structures that dominate the last stage of transition. Spots production affects the length of transition region. The turbulent spots creation rate, growth characteristics and their merger lead to fully developed turbulent flow. A brief summary on turbulent spot and calmed region was compiled in Jonáš [3].

The presence of hairpin shaped vortical structures in boundary layers during transition process to turbulence has been postulated and pursued by a number of investigators over the past half century since the original work of Theodorsen [4]. The availability of direct and large eddy numerical simulations in the 1980s provided more direct and statistical evidence in support of the presence of these structures in turbulent shear ows. Interestingly, vortical structures extracted by Robinson [5] from Spalart's direct numerical simulation database of a modeled boundary layer were not consistent with the postulated dominance of hairpin structures. However, none of the previous simulations were of a genuine spatially developing turbulent boundary layer. Hairpin packets arising from upstream fragmented structures are found to be instrumental in the breakdown of the boundary layer bypass transition.

\footnotetext{
- Ing. Ondřej Hladík: Institute of Thermomechanics, AS CR, v.v.i., Dolejškova 5, 18200 Praha 8; CZ, e-mail: hladik@it.cas.cz

- RNDr. Pavel Jonáš, DrSc.: Institute of Thermomechanics, AS CR, v.v.i., Dolejškova 5, 18200 Praha 8; CZ, e-mail: jonas@it.cas.cz

- Doc. Ing. Václav Uruba, CSc.: Institute of Thermomechanics, AS CR, v.v.i., Dolejškova 5, 18200 Praha 8; CZ, e-mail: uruba@it.cas.cz
} 
The velocity fluctuations inside a spot have turbulent nature with high frequency fluctuation and with increasing both the streamwise velocity component and the wall shear stress as the spot is passing a point (fixed probe). Turbulence dissipation occurs almost exclusively in this zone. A calmed region is attached at the rear of a turbulent spot in a laminar or transitional boundary layer. Existence of the calmed region have been proved in photos in Cantwell, Coles \& Dimotakis [6], which show the presence of streaky structures inside. Jacobs \& Durbin [7] performed DNS simulation of such a type of flow, which proved that long streaks of streamwise velocity perturbation described above were initiated by low-frequency modes from the free-stream.

Visualizations of the structure of both transitional and fully turbulent boundary layers are shown e.g. in Wu \& Moin [8], the data was generated using DNS method. The PIV method applied to the plains parallel to the wall has been used for study of the transitional boundary layer structure was suggested for the first time in Longmire, Ganapathisubramani, Marusic, Urness \& Interrante [9].

\section{EXPERIMENTAL SETUP}

The experiments have been carried out in the blow-down facility with rectangular crosssection $250 \times 100 \mathrm{~mm}^{2}$ and 3 meters in length. Velocity of the air-flow was about $4.6 \mathrm{~m} / \mathrm{s}$ in the channel inlet, the top-hat profile was with intensity of fluctuations less than $0.1 \%$ and the deviations of the mean velocity were less than $1 \%$ throughout the cross-section. Experimental study of dynamics of the turbulent structures is rather complicated, because both temporal and spatial correlations should be taken into account simultaneously. That is why the time-resolved PIV technique has been used. The DANTEC system consists of laser with cylindrical optics, the CMOS camera and software Dynamics Studio 3.14. Laser New Wave Pegasus Nd:YLF, double head, wavelength $527 \mathrm{~nm}$, maximal frequency $10 \mathrm{kHz}$, a shot energy is $10 \mathrm{~mJ}$ for $1 \mathrm{kHz}$ (corresponding power $10 \mathrm{~W}$ per head). The camera NanoSense MkIII, maximal resolution $1280 \times 1024$ pixels and corresponding maximal frequency 500 double-snaps per second. For particle generation the fog-generator SAFEX is used. The velocity has been evaluated in the grid $63 \times 79$ interrogation area $32 \times 32$ pixels, overlap $50 \%$. The 1500 subsequent complete vector fields are evaluated with frequency $500 \mathrm{~Hz}$ representing $3 \mathrm{~s}$ in physical time.

The standard Cartesian coordinate system $x y z$ has been introduced with $x$ streamwise direction and $y$ direction perpendicular to the wall. Two configurations were used in experiments.

First, the velocity measurements were performed in the xy plane perpendicular to the wall, secondly the measuring plane was the $x z$ plane parallel to the wall. Then the distance of the measuring plane from wall was $y \approx 3 \mathrm{~mm}$. Please note that the thickness of the laser sheet is about $1 \mathrm{~mm}$.

\section{RESULTS}

First, the measurements of the boundary layer structure in plane perpendicular to the wall oriented in the streamwise direction were performed. The boundary layers on the channel walls are subjected to laminar-turbulent transition process starting at position $x \approx 800 \mathrm{~mm}$ while in position $x=2500 \mathrm{~mm}$ we observed nearly fully developed turbulent boundary layer, which could be characterized by the conventional (half-per-cent) boundary layer thickness $\delta_{0.995}=22.5 \mathrm{~mm}$ the shape factor $H_{12}=1.53$. We decided to perform our experiments in position $x=1200 \mathrm{~mm}$ corresponding Reynolds number based 
on distance from the boundary layer origin $\mathrm{Re}_{x}=1.4 \cdot 10^{5}$. In this position the turbulent spots appeared intermittently, so we could conclude that the last stage of the transition process is running there. This statement is supported by the fact, that the shape factor $H_{12}=2.24$, conventional boundary layer thickness was $\delta_{0.995}=12.05 \mathrm{~mm}$ and impulse thickness is about $1.5 \mathrm{~mm}$ resulting in $\mathrm{Re}_{\theta}=430$. We shifted the origin of the coordinate system to be $1200 \mathrm{~mm}$ downstream from the channel inlet, on the wall middle.

For topology study the mean velocity (oriented from left to right) is subtracted from each instantaneous velocity field defining thus the velocity fluctuations time series. In the velocity fields the distribution of velocity vectors fluctuations is presented. The unity vector is given in left-top corner as a scale. In colour calculated vorticity with direction orthogonal to the measuring plane is presented, red colour denotes positive values and blue negative. The green lines are isolines of instantaneous longitudinal velocity fluctuation component ( $x$ axis direction), however only areas of negative values are depicted. This is to enable recognition of the low-velocity streaks within the flow-field.

In Fig. 1 there are examples of situations when a turbulent spot is present in the flowfield, while Fig. 2 represents laminar state between the spots. Fig. 3 shows a spot rearparts with calmed region.
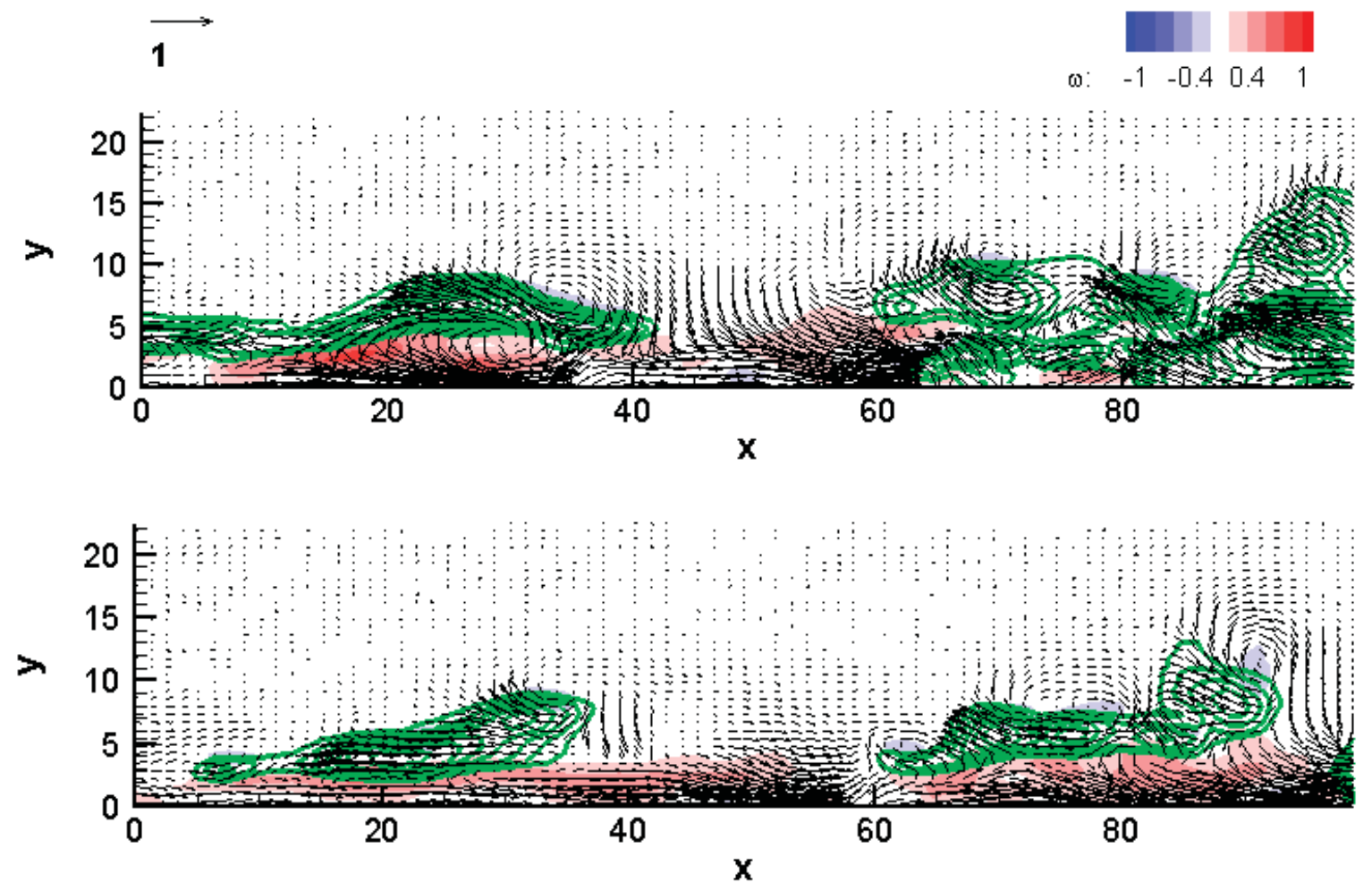

Figure 1: Two different turbulent spots in side view.

Fig. 1 reveals complicated fractal structure of a turbulent spot with structures of different sizes. The negative velocity fluctuations are sitting on the top of positive vorticity regions.

The laminar phase in Fig. 2 is characterized by minimal fluctuations, as a rule. However there are regions of low-velocity located close to the wall indicating a different instantaneous velocity profile with lower skin-friction corresponding to Blasius-type profile. 


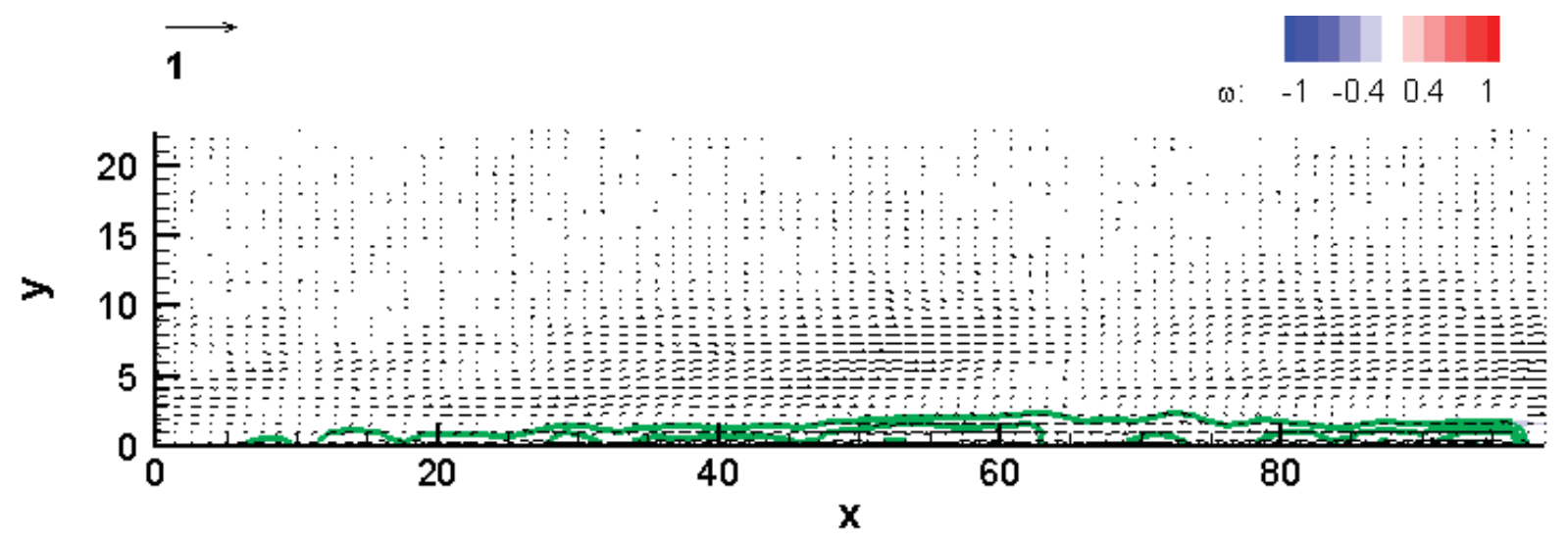

Figure 2: Side view on laminar phase.

The laminar phase in Fig. 2 is characterized by minimal fluctuations, as a rule. However there are regions of low-velocity located close to the wall indicating a different instantaneous velocity profile with lower skin-friction corresponding to Blasius-type profile.

In Fig. 3 the rear-parts of a turbulent spots are shown with their ends on positions $x \approx 60$ and $x \approx 80$. In the calmed region we could recognize the zone of acceleration (i.e. positive longitudinal velocity fluctuation) forming the flow behind the spot.
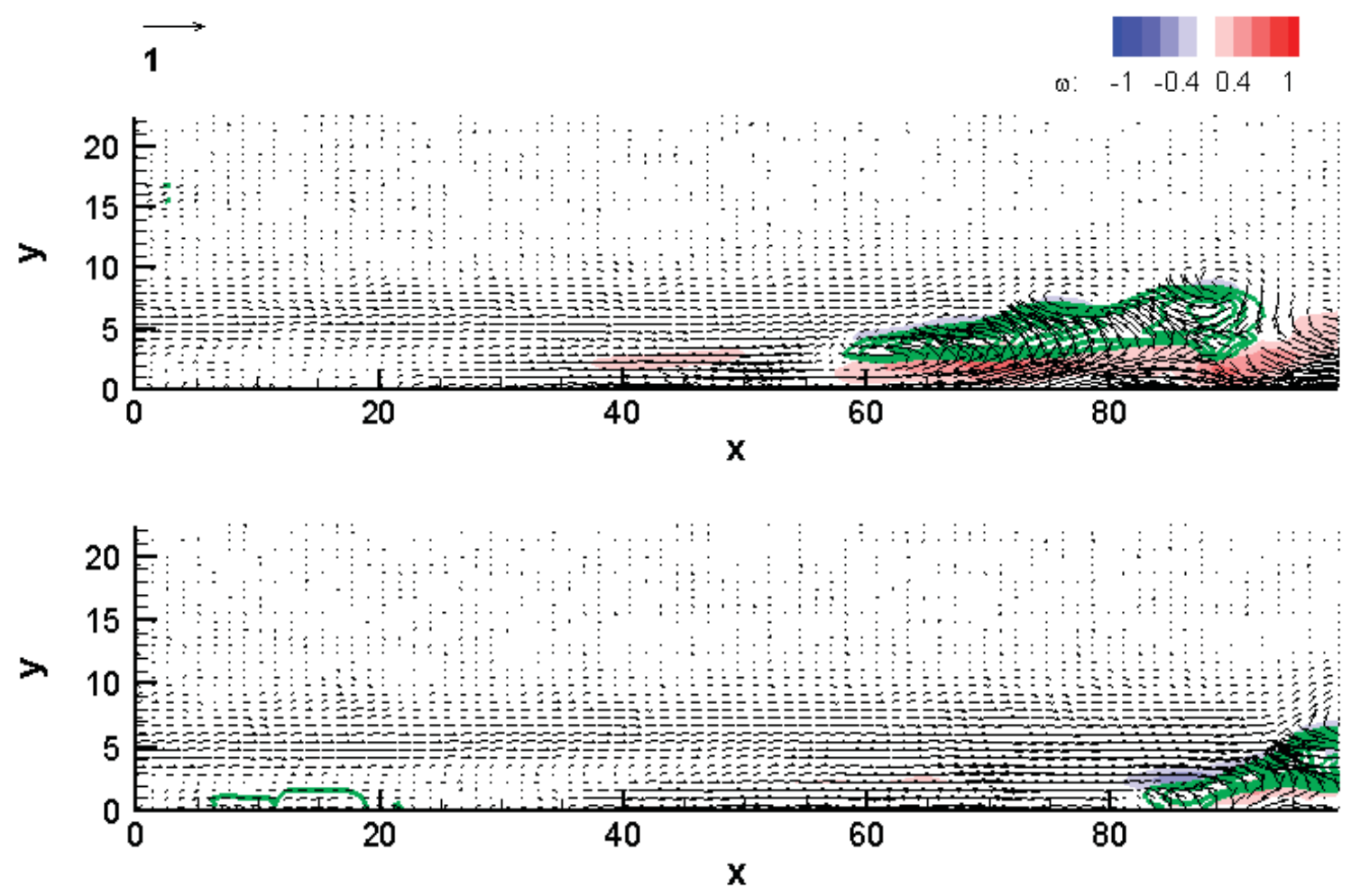

Figure 3: Two different calmed regions in side view. 
Then, the measurements in the plane parallel to the wall have been carried out. The mean velocity field corresponding to approximately constant value throughout the investigated region and it was subtracted from all instantaneous velocity distributions. The structure of the flow-field changed intermittently.

The packets of hairpin vortices are identified using the strategy presented in Longmire, Ganapathisubramani, Marusic, Urness \& Interrante [9]. Supposing mean flow from left to the right, a hairpin vortex is characterized by negative vorticity on the top of the region, positive on the bottom and negative longitudinal velocity area in between forming well known low-velocity streaks.

In Fig. 4 the top views on structures within a turbulent spot is shown. Fig. 5 shows typical situation in laminar state, while Figs. 6 and 7 represent structures in the spot rear-parts and in the calmed region, respectively.
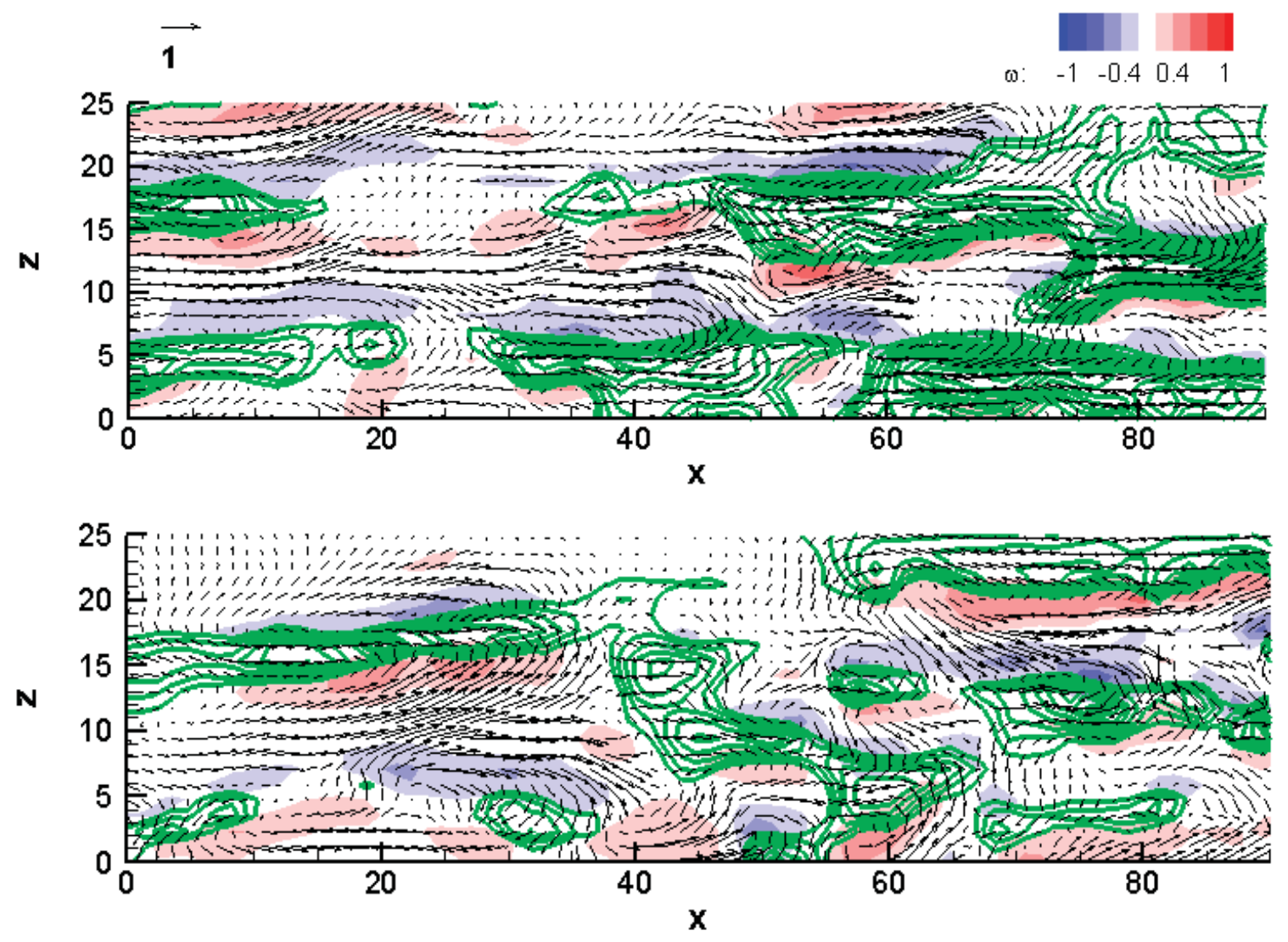

Figure 4: Two different turbulent spots in top view.

In a turbulent spot Fig. 4 typical configurations of negative vorticity on the top, negative vorticity on the bottom and low velocity region in between could be recognized forming hairpin vortices packets oriented in streamwise direction. Various sizes of the structures indicate fractal structure of hairpins.

The laminar phase Fig. 5 is characterized by low-velocity regions without vortical structures. The calmed region behind a turbulent spot in Fig. 6 shows distinct longitudinal streaks of low-velocity (i.e. negative fluctuation) located in certain z positions. 


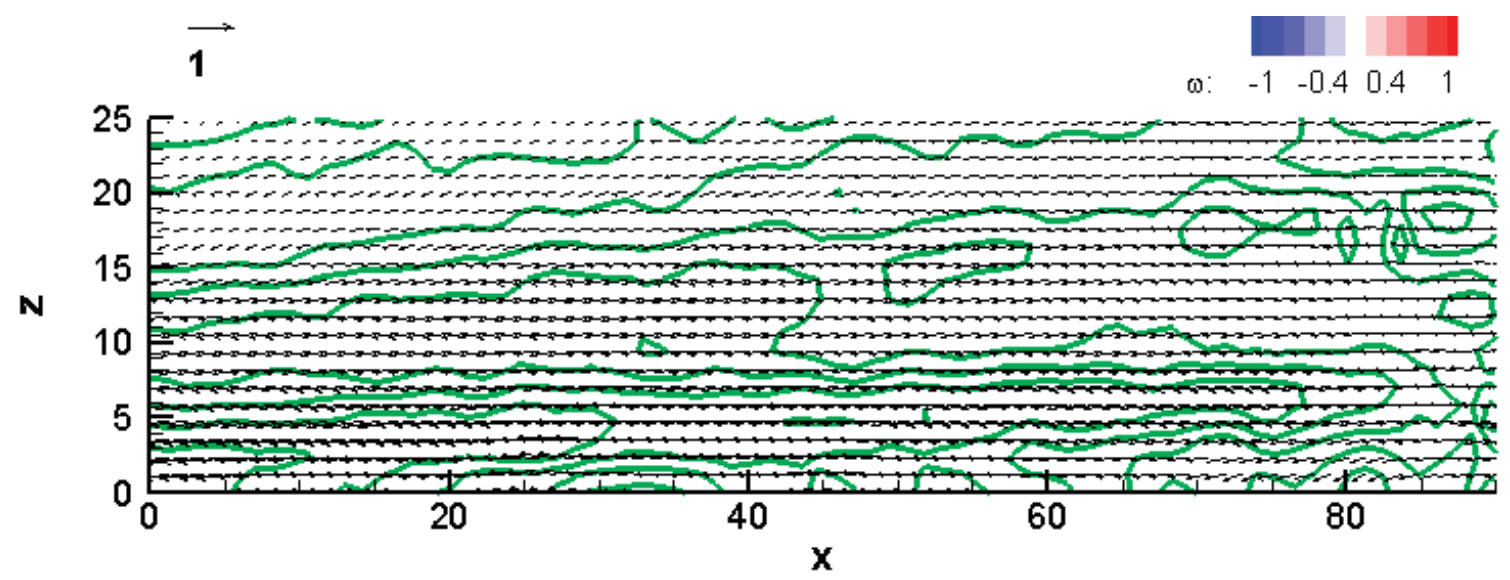

Figure 5: Top view on laminar phase.

Apparently, the situation in Fig. 3 corresponds to position between the streaks, as positive fluctuation is detected. The low-velocity streaks origin is typically between the elongated vortical structures in the spots rear-part. The elongated vortical structures could be relatively long in streamwise direction as visible in Fig. 7, however the configuration in this figure generates no streak, because the vorticity orientation order is reverse here (positive on top and negative on bottom).
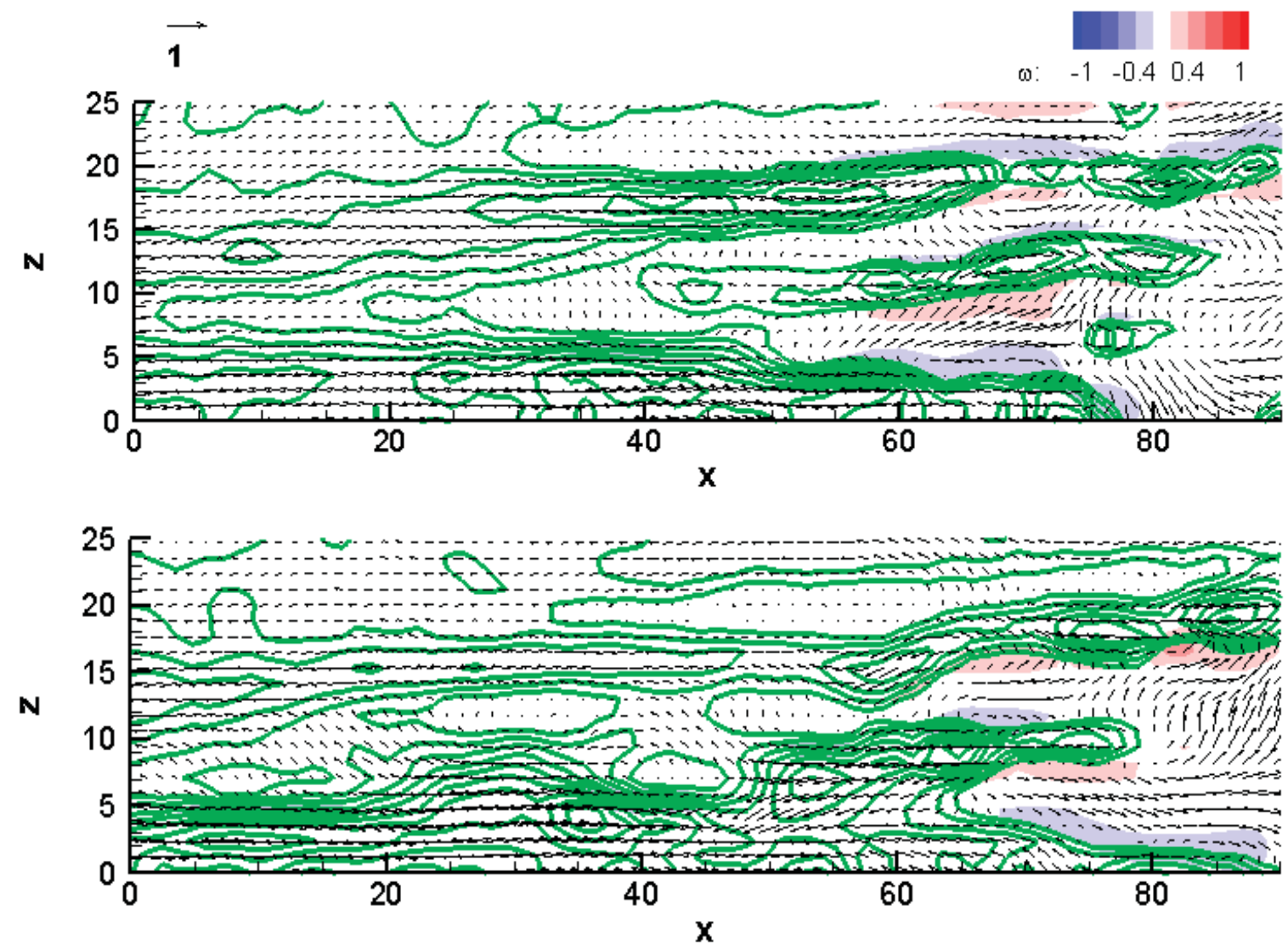

Figure 6: Two different calmed regions in top view. 


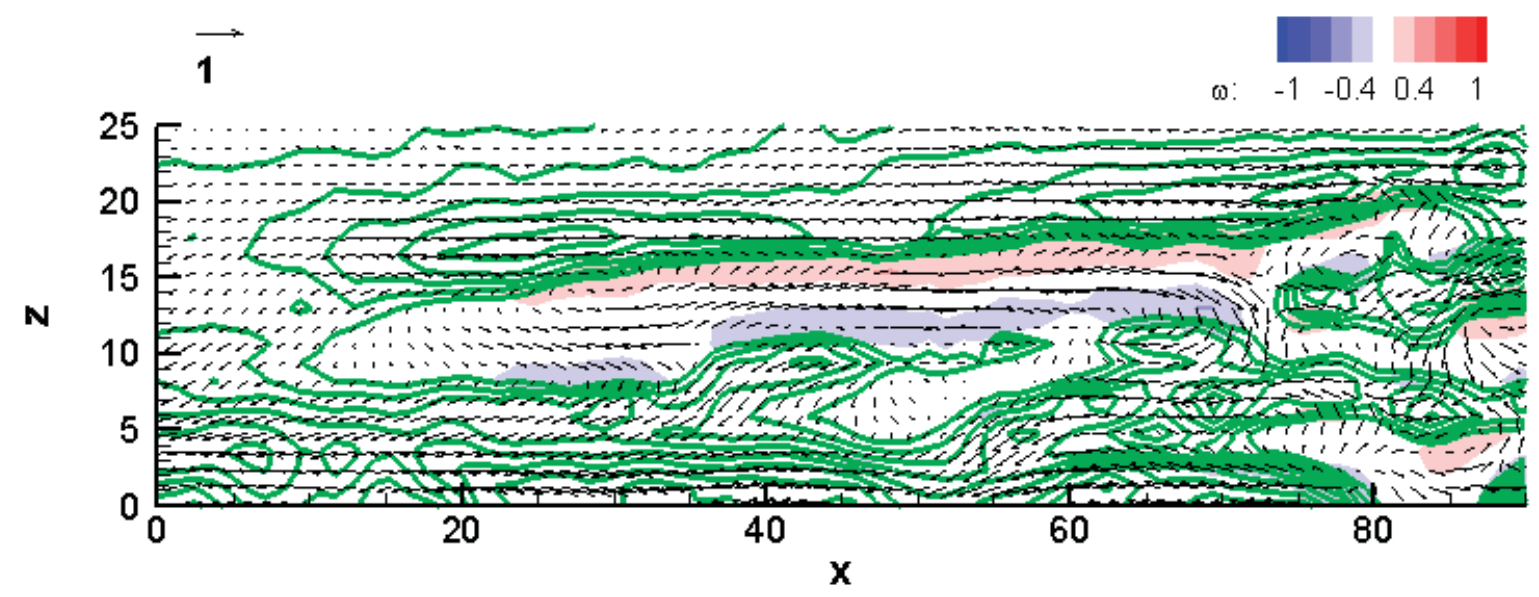

Figure 7: Top view on elongated vortical structures.

\section{CONCLUSION}

The transitional boundary layer was subjected to experiments using time-resolved PIV technique. The boundary layer was in the last stage of transition process which is characterized by intermittent turbulent spots appearance. The hairpin vortices and packets of hairpin vortices were identified within turbulent spots.

\section{Acknowledgments}

The author gratefully acknowledges financial support of the Grant Agency of the Czech Republic, projects No. $101 / 08 / 1112$ and $\mathrm{P} 101 / 10 / 1230$ as well as the project AVOZ20760514.

\section{REFERENCES}

[1] Emmons, H. W.: The Laminar-Turbulent Transition in Boundary Layers. J. Aeronaut. Sci. 18, 1951, 490-498.

[2] Narasimha, R..The laminar-turbulent transition zone in the boundary layer. Progress in Aerospace Science 22, 1985 . 29-80.

[3] Jonáš, P.: On the turbulent spot and calmed region. In Engnr. Mech. 2007, ed. I. Zolotarev, IT AS CR Praha, 2007, 109-110.

[4] Theodorsen, T.:Mechanism of turbulence. In Proc. 2nd Midwestern Conf. on Fluid Mech., Columbus, Ohio, Ohio State Univ., 1952,1-19.

[5] Robinson, S. K.: Coherent motion in the turbulent boundary layer. Annu. Rev. Fluid Mech. 23, 1991, 601-639.

[6] Cantwell, B., Coles, D. \& Dimotakis, P.: Structure and entrainment in the plane of symmetry of a turbulent spot. J. Fluid. Mech. 87, 1978. 641-672.

[7] Jacobs R. G. \& Durbin P. A.: Simulations of bypass transition. J. Fluid Mech. 428, 2001. 185-212.

[8] Wu, X. \& Moin, P.: Forest of hairpins in a low-Reynolds-number zero-pressuregradient flat-plate boundary layer. Phys. Fluids 21, 2009, 091106.

[9] Longmire, E. K., Ganapathisubramani, B., Marusic, I., Urness, T., \& Interrante, V.: Effective visualization od stereo PIV vector fields of turbulent boundary layer. Journal of Turbulence, No 23 4, 2003, 1468-5248. 
[10] Adrian, R. J., Meinhart, C. D. \& Tomkins C. D.: Vortex organization in the outer region of the turbulent boundary layer. J. Fluid Mech. 2000 , 422, 1-54.

[11] Head, M. R. \& Bandyopadhya,y P.: New aspects of turbulent boundary layer structure. J. Fluid Mech. 107, 1981, 297-338.

[12] Jonáš, P., Mazur, O. \& Uruba, V.: On the receptivity of the by-pass transition to the length scale of the outer stream turbulence. Eur.J.Mech. B 19, 2000, 707722.

[13] Jonáš, P., Mazur, O. \& Uruba, V.: Comparison between the wall roughness effect and the free stream turbulence impact and their joint action on a boundary layer development. ERCOFTAC Bulletin 80, 2009, 82-86.

[14] Jonáš, P., Elsner, W., Mazur, O., Uruba, V. \& Wysocki, M.: Turbulent spots detection during boundary layer by-pass transition. ERCOFTAC Bulletin 80, 2009, 16-19.

[15] Panton, R. L.: Overview of the self-sustaining mechanisms of wall turbulence. Prog. Aerospace Sci. 37, 200, 341-383. 